\title{
Estimating the Lifetime of the Pneumatic Cylinder in Machine Tool Subjected to Repetitive Pressure Loading
}

\author{
Seong-woo Woo \\ Reliability Association of Korea, Seoul, Republic of Korea
}

\begin{abstract}
To estimate the lifetime of pneumatic cylinder in machine tool (or automatic assembly line), new reliability methodology for parametric accelerated life testing (ALT) is suggested. It consists of parametric ALT plan, generalized life-stress failure model with a new effort concept, acceleration factor, and sample size equation. This new parametric ALT enables an engineer to estimate the lifetime of a commercially parts by uncovering the failure through accelerated testing under severe conditions. As a case study, two commercial products of the pneumatic cylinder in machine tool were tested. For company A, we found the hardening and wear of piston seal like that of field at normal conditions. As increasing the pressure, the pneumatic cylinders were tested. Based on the accelerated conditions-0.8 MPa and $1.2 \mathrm{MPa}$ at $23^{\circ} \mathrm{C}$, B1 life at $0.63 \mathrm{MPa}$ and $23^{\circ} \mathrm{C}$ was extrapolated as $2.39 \times$ $10^{6}$. On the other hand, for company $\mathrm{B}$, we found the rod cap blocked by slurry like those of field samples. Based on the accelerated conditions- $-8 \mathrm{MPa}$ and $1.4 \mathrm{MPa}$ at $23^{\circ} \mathrm{C}$, B1 life at $0.63 \mathrm{MPa}$ and $23^{\circ} \mathrm{C}$ was extrapolated as $2.51 \times 10^{6}$. Comparing them with testing data at normal use conditions, we knew that the estimation error for both companies was less than $10 \%$.
\end{abstract}

Keywords: reliability design, lifetime, parametric accelerated life testing, pneumatic cylinder system

\section{Nomenclature}

Area, $\mathrm{m}^{2}$

Acceleration factor

Durability index

Activation energy

Unreliability

Testing cycles (or cycles)

Non-dimensional testing cycles, $h^{*}=h / L_{B} \geq 1$

Reaction rate

Target $B X$ life and $x=0.01 \mathrm{X}$, on the condition that $x \leq 0.2$

Number of test samples

Energy, $k J$

Failed numbers

Reliability

Stress

Mechanical stress under accelerated stress conditions

Corresponding author: Seong-woo Woo, Ph.D., mechanical engineer, Reliability Association of Korea, Seoul, Republic of Korea; research fields: computational or experimental thermo-fluid sciences or advanced energy systems, reliability design for electronic and mechanical components. 
$S_{0}$

$t_{i}$

$T F$

$x$

Greek Symbols

$\eta$

$\lambda$

\section{Superscripts}

$\beta$

$n$

\section{Subscripts}

0

1
Mechanical stress under normal conditions

Test time for each sample, $h$

Time to failure, $h$

Required target $x=0.01 \cdot X$, on condition that $x \leq 0.2$.

\section{Characteristic life}

Cumulative damage exponent

Shape parameter in a Weibull distribution

Stress dependence, $n=-\left[\frac{\partial \ln \left(T_{f}\right)}{\partial \ln (S)}\right]_{T}$

Normal stress conditions

Accelerated stress conditions

\section{Introduction}

The pneumatic cylinder is widely a key mechanical component that is used in the tool changing device of machine tool or the automatic assembly line in the flexible manufacturing system. As the pneumatic cylinder in field is repetitively subjected to different pressure loads, manufacturers of pneumatic components supply their lifetime to end-users in accordance with ISO Standard (International Organization for Standardization, 2007).

For instance, a machine tool is to shape machining metal or other rigid materials by cutting, boring, grinding, shearing, or other forms of deformation. Machine tool including pneumatic cylinder can consist of several different modules - tool (or pellet) changing device, spindle/drive unit, hydropower unit, tilting index table, turret head, cooler unit, and CNC controller (see Figure 1).

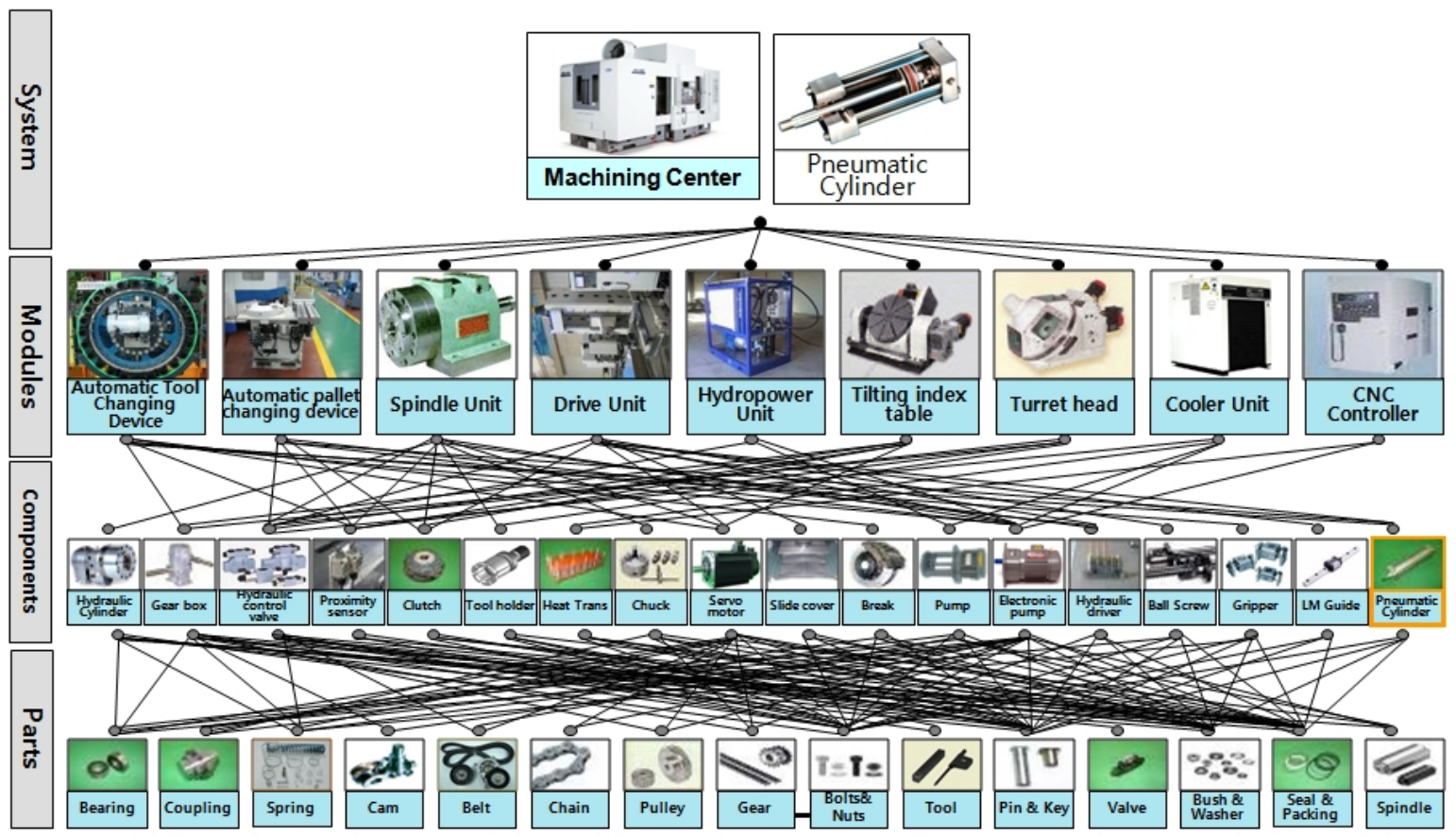

Figure 1. Breakdown of a machine tool with its modules, components, and parts. 
A tool-changing device, key module in a machine tool, is selectively presenting a desired tool to the work piece. For this (intended) function, the pneumatic cylinder uses compressed air acting on a piston inside a cylinder to move a load along a linear path. Pneumatic cylinder therefore is designed to secure the lifetime specified by the end-users who purchase and use the machine tool. If not, the manufacturing system will stop abruptly.

To respond the field demand, the pneumatic cylinder might be replaced as new one before reaching its lifetime. If so, end-users properly manufacture their commodity and prevent big damages from the failures of pneumatic cylinder in field. Proper reliability testing is required to achieve whether the design of pneumatic cylinder is adequate to meet the requirements of reliability. It enables end-users to estimate the lifetime of pneumatic cylinder (Ben-Gal, Herer, \& Raz, 2003; Nelson, 1980; Spencer, 1991; Elsayed, 2003).

However, there are pending questions_-assigning reliability target, accelerated testing, and sample size equation —in reliability testing. If fewer samples are selected, the statistical data accuracy for reliability testing will decrease. If a sufficient quantity of parts is tested, the cost and time will demand considerably. Therefore, the best solution that can decrease the sample size and increase the data accuracy is the accelerated life testing (ALT) based on the load analysis. Parametric ALT can save the testing time and decrease sample size under accelerated conditions (Woo \& Pecht, 2008; Woo, O’Neal, \& Pecht, 2009a; 2009b; 2009c; 2010a; 2010b; 2011; Woo \& O’Neal, 2015; Woo, 2017). It will expose the weakest parts in product due to design problems. Engineer ultimately can estimate the product lifetime.

The purpose of this study is to present the reliability methodology that estimates the lifetime of the mechanical system subjected to repetitive loading like commercially produced machine tools. The reliability methodology included: (1) parametric ALT plan; (2) load analysis; (3) a tailored sample of parametric ALTs; and (4) estimation of the lifetime of the mechanical parts. If the commercially designed mechanical system is operated to the mission cycle, the reliability target will be achieved. As case study, we will test the pneumatic cylinder produced by two commercial companies and verify their effectiveness.

\section{Materials \& Methods}

\section{Setting Overall Parametric ALT Plan of Mechanical Product}

Reliability of mechanical system can be defined as the ability of a system or module to function under stated conditions for a specified period of time (Standards Coordinating Committee of the Computer Society of IEEE, 1990). It can be illustrated in diagram called "the bathtub curve" that consists of three parts. First, there is a decreasing failure rate, then a constant failure rate, and then an increasing failure rate. If a product follows the bathtub curve, it will have difficulties in succeeding in the market. Because of the faulty design, the mechanical product will have higher failure rates in operation and incur financial losses for the company. The company will then need to set goals for new products to: (1) reduce early failures; (2) decrease random failures during the product operating time; and (3) increase product lifetime. As the reliability of a mechanical product is improved, the failure rate of the product in the field will decline. For such a situation, the traditional bathtub curve can be transformed to a straight line with the shape parameter $\beta$ (see Figure 2).

Finally, reliability of the mechanical product might be quantified from the product lifetime $L_{B}$ and failure rate $\lambda$ as follows:

$$
\mathrm{R}\left(\mathrm{L}_{\mathrm{B}}\right)=1-F\left(L_{B}\right)=\mathrm{e}^{-\lambda \mathrm{L}_{\mathrm{B}}} \cong 1-\lambda \mathrm{L}_{\mathrm{BX}}
$$




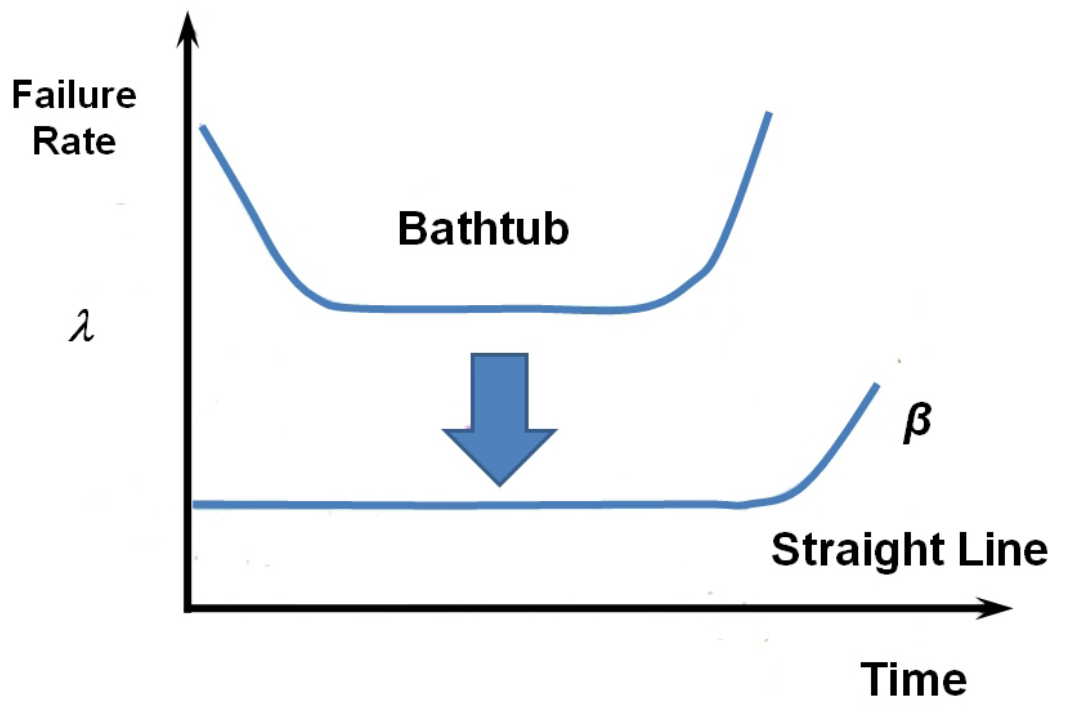

Figure 2. Bathtub curve and straight line with slope $\beta$.

This equation is applicable below about 20 percent of cumulative failure (Kreyszig, 2006). Reliability design of the mechanical system can be achieved by getting the targeted product lifetime $L_{B}$ and failure rate $\lambda$ after finding the missing control parameters and modifying the defective configuration of structures through parametric accelerated life testing (ALT).

Table 1

Overall Parametric ALT Plan of Machine Tools

\begin{tabular}{llllcllll}
\hline \multirow{2}{*}{ Modules } & \multicolumn{3}{c}{ Market data } & \multicolumn{3}{c}{ Expected reliability } & \multicolumn{2}{c}{ Targeted reliability } \\
\cline { 2 - 9 } & $\begin{array}{l}\text { Yearly failure } \\
\text { rate, \%/yr }\end{array}$ & $B x$ life, yr & \multicolumn{3}{c}{$\begin{array}{c}\text { Yearly failure rate, } \\
\% / y r\end{array}$} & $B_{x}$ life, yr & $\begin{array}{l}\text { Yearly failure } \\
\text { rate, \%/yr }\end{array}$ & $\begin{array}{l}B x \text { life, } \\
\text { yr }\end{array}$ \\
\hline A & 0.35 & 2.9 & Similar & $\times 1$ & 0.35 & 2.86 & 0.10 & $10(x=1.0)$ \\
B & 0.24 & 4.2 & New & $\times 5$ & 1.20 & 0.83 & 0.15 & $10(x=1.5)$ \\
C & 0.30 & 3.3 & Similar & $\times 1$ & 0.30 & 3.33 & 0.10 & $10(x=1.0)$ \\
D & 0.31 & 3.2 & Modified & $\times 2$ & 0.62 & 1.61 & 0.10 & $10(x=1.0)$ \\
E & 0.15 & 6.7 & Modified & $\times 2$ & 0.30 & 3.33 & 0.15 & $10(x=1.5)$ \\
Others & 0.50 & 2.0 & Similar & $\times 1$ & 0.50 & 2.00 & 0.40 & $10(x=4.0)$ \\
Product (Total) & 1.9 & 5.4 & - & - & 3.27 & 3.06 & 1.00 & $10(x=10)$ \\
\hline
\end{tabular}

In targeting the reliability of the mechanical product in parametric ALT, there are three cases for modules in mechanical product: (1) a modified module; (2) new module; and (3) similar module to the prior design on the basis of market data. Tool changing device module including pneumatic device is the module modified due to the cost reduction.

Like Module D listed in Table 1, tool changing device from the field data was that the yearly failure rate was $0.62 \% / y e a r$ and lifetime, $L_{B 1}$, was 1.61 years. To respond a customer claims, new target for tool changing device including pneumatic cylinder was set to be B1 of 10 years with 1.0 yearly failure rate.

\section{Parametric Accelerated Life Testing of Mechanical System}

Acceleration Factor (AF). For solid-state diffusion of impurities in silicon, the junction equation $J$ might be expressed as: 


$$
\begin{gathered}
J=[a C(x-a)] \cdot \exp \left[-\frac{q}{k T}\left(w-\frac{1}{2} a \xi\right)\right] \cdot v \\
=-\left[a^{2} v e^{-q w / k T}\right] \cdot \cosh \frac{q a \xi}{2 k T} \frac{\partial C}{\partial x}+\left[2 a v e^{-q w / k T}\right] C \sinh \frac{q a \xi}{2 k T} \\
=\Phi(x, t, T) \sinh (a \xi) \exp \left(-\frac{Q}{k T}\right) \\
=A \sinh (a \xi) \exp \left(-\frac{Q}{k T}\right)
\end{gathered}
$$

On the other hand, reaction process that is dependent to speed might be expressed as:

$$
\begin{aligned}
K & =K^{+}-K^{-}=a \frac{k T}{h} e^{-\frac{\Delta E-a S}{k T}}-a \frac{k T}{h} e^{-\frac{\Delta E+a S}{k T}} \\
& =a \frac{k T}{h} e^{-\frac{\Delta E}{k T}} \sinh \left(\frac{a S}{k T}\right)
\end{aligned}
$$

So, the reaction rate $K$ from equation (2) and (3) can be summarized as:

$$
K=B \sinh (a S) \exp \left(-\frac{E_{a}}{k T}\right)
$$

If the reaction rate in equation (4) takes an inverse number, the generalized stress model can be obtained as:

$$
T F=A[\sinh (a S)]^{-1} \exp \left(\frac{E_{a}}{k T}\right)
$$

As seen in Figure 3, the range of the hyperbolic sine stress term $[\sinh (a S)]$ in equation (5) is increasing the stress as following: (1) initially $(S)$ in low effect; (2) $(S)^{n}$ in medium effect; and (3) $\left(e^{a S}\right)^{n}$ in high effect initially linearly increasing. Accelerated testing usually happens in the medium stress range.

Thus, time to failure in the level of medium stress can then be described as:

$$
T F=A(S)^{-n} \exp \left(\frac{E_{a}}{k T}\right)
$$

The internal (or external) stress in a product is difficult to quantify and use in accelerated testing. It is necessary to modify equation (6) into a more applicable form. The power in a mechanical system can be expressed as efforts and flows (Karnopp, Margolis, \& Rosenberg, 2000):

$$
P=e(t) \cdot f(t)
$$




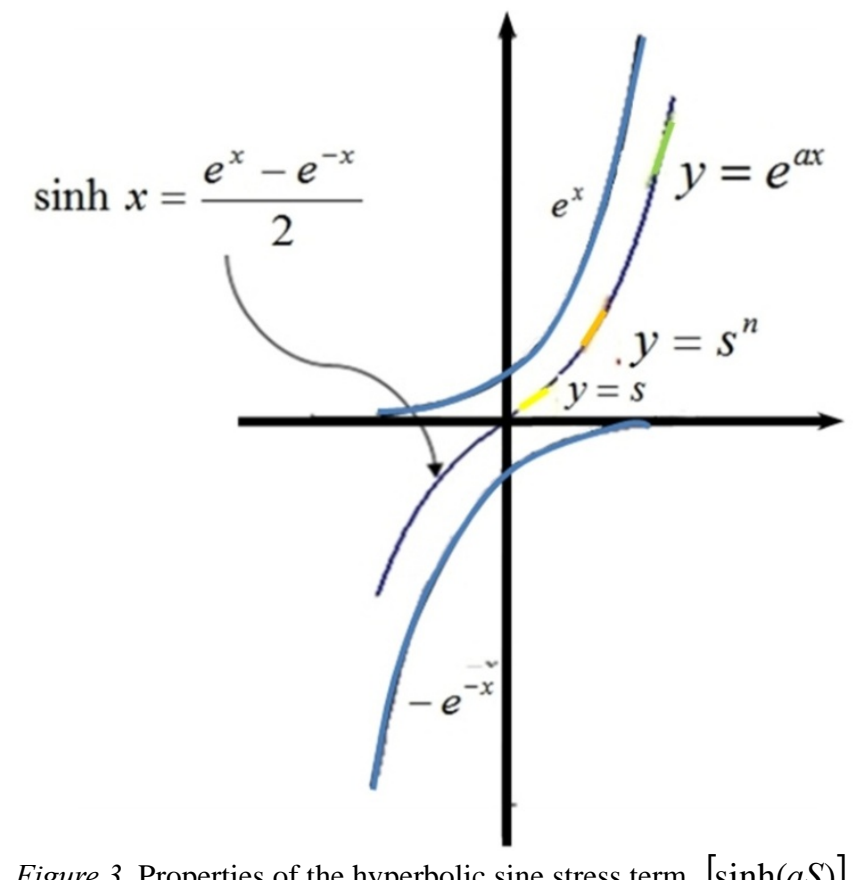

Thus, stresses in mechanical systems may come from the efforts (or loads) like force, torque, and pressure. For a mechanical system, when replacing stress with effort, the time-to-failure can be modified as:

$$
T F=A(S)^{-n} \exp \left(\frac{E_{a}}{k T}\right)=A(e)^{-\lambda} \exp \left(\frac{E_{a}}{k T}\right)
$$

From the time-to-failure in equation (8), the acceleration factor can be defined as the ratio between the proper accelerated stress levels and typical operating conditions. The acceleration factor (AF) can be modified to include the effort concepts:

$$
A F=\left(\frac{S_{1}}{S_{0}}\right)^{n}\left[\frac{E_{a}}{k}\left(\frac{1}{T_{0}}-\frac{1}{T_{1}}\right)\right]=\left(\frac{e_{1}}{e_{0}}\right)^{\lambda}\left[\frac{E_{a}}{k}\left(\frac{1}{T_{0}}-\frac{1}{T_{1}}\right)\right]
$$

Derivation of sample size equation. To derive the sample size equation and carry out a parametric ALT, the characteristic life $\eta$ needed to be estimated from the Weibull distribution. First, the characteristic life $\eta_{M L E}$ from the Maximum Likelihood Estimation (MLE) was derived as:

$$
\eta_{M L E}^{\beta}=\sum_{i=1}^{n} \frac{t_{i}^{\beta}}{r}
$$

If the confidence level was 100(1- $\alpha$ ) and the number of failures was $r \geq 1$, the characteristic life, $\eta_{\alpha}$, could be estimated from equation (10):

$$
\eta_{\alpha}^{\beta}=\frac{2 r}{\chi_{\alpha}^{2}(2 r+2)} \cdot \eta_{M L E}^{\beta}=\frac{2}{\chi_{\alpha}^{2}(2 r+2)} \cdot \sum_{i=1}^{n} t_{i}^{\beta} \quad \text { for } r \geq 1
$$

If there was no failures, the $p$-value was $\alpha$ and $\ln (1 / \alpha)$ was mathematically equivalent to the Chi-square 
value, $\frac{\chi_{\alpha}^{2}(2)}{2}$. The characteristic life $\eta_{\alpha}$ would be expressed as:

$$
\eta_{\alpha}^{\beta}=\frac{2}{\chi_{\alpha}^{2}(2)} \cdot \sum_{i=1}^{n} t_{i}^{\beta}=\frac{1}{\ln \frac{1}{\alpha}} \cdot \sum_{i=1}^{n} t_{i}^{\beta}, \quad \text { for } r=0
$$

Because equation (11) was established for all cases $r \geq 0$, it can be redefined as:

$$
\eta_{\alpha}^{\beta}=\frac{2}{\chi_{\alpha}^{2}(2 r+2)} \cdot \sum_{i=1}^{n} t_{i}^{\beta} \quad \text { for } r \geq 0
$$

The Cumulative Distribution Function (CDF) in the Weibull function can be expressed as:

$$
F(t)=1-e^{-\left(\frac{t}{\eta}\right)^{\beta}}
$$

To evaluate the Weibull reliability function, the characteristic life can be converted into $L_{B X}$ life as follows:

$$
R(t)=e^{-\left(\frac{L_{B X}}{\eta}\right)^{\beta}}=1-x
$$

After logarithmic transformation, equation (15) can be expressed as:

$$
L_{B X}^{\beta}=\left(\ln \frac{1}{1-x}\right) \cdot \eta^{\beta}
$$

If the estimated characteristic life of $p$-value $\alpha, \eta_{\alpha}$, in equation (13), was substituted into equation (11), we obtain the $B_{X}$ life equation:

$$
L_{B X}^{\beta}=\frac{2}{\chi_{\alpha}^{2}(2 r+2)} \cdot\left(\ln \frac{1}{1-x}\right) \cdot \sum_{i=1}^{n} t_{i}^{\beta}
$$

If the sample size was large enough, the planned testing time should proceed as:

$$
\sum_{i=1}^{n} t_{i}^{\beta} \cong n \cdot h^{\beta}
$$

The estimated lifetime $\left(L_{B X}\right)$ in test should be longer than the targeted lifetime $\left(L^{*}{ }_{B X}\right)$ :

$$
L_{B X}^{\beta} \cong \frac{2}{\chi_{\alpha}^{2}(2 r+2)} \cdot\left(\ln \frac{1}{1-x}\right) \cdot n h^{\beta} \geq L_{B X}^{* \beta}
$$

Then, sample size equation is expressed as follows:

$$
n \geq \frac{\chi_{\alpha}^{2}(2 r+2)}{2} \cdot \frac{1}{\left(\ln \frac{1}{1-x}\right)} \cdot\left(\frac{L_{B X}^{*}}{h}\right)^{\beta}
$$


However, most of the lifetime testing had insufficient samples. The allowed number of failures would not have as much as that of the sample size:

$$
\sum_{i=1}^{n} t_{i}^{\beta}=\sum_{i=1}^{r} t_{i}^{\beta}+(n-r) h^{\beta} \geq(n-r) h^{\beta}
$$

If equation (21) was substituted into equation (17), $B X$ life equation can be modified as follows:

$$
L_{B X}^{\beta} \geq \frac{2}{\chi_{\alpha}^{2}(2 r+2)} \cdot\left(\ln \frac{1}{1-x}\right) \cdot(n-r) h^{\beta} \geq L_{B X}^{* \beta}
$$

Then, the sample size equation with the number of failure can also be modified as:

$$
n \geq \frac{\chi_{\alpha}^{2}(2 r+2)}{2} \cdot \frac{1}{\left(\ln \frac{1}{1-x}\right)} \cdot\left(\frac{L_{B X}^{*}}{h}\right)^{\beta}+r
$$

From the sample size equation (23), we can carry out with lifetime testing (or parametric ALT testing) under any failure conditions $(r \geq 0)$. Consequently, it also confirmed whether the failure mechanism and the test method were proper.

For a 60\% confidence level, the first term $\frac{\chi_{\alpha}^{2}(2 r+2)}{2}$ in equation (23) can be approximated as $(r+1)$ (Ryu $\&$ Chang, 2005). If the cumulative failure rate, $x$, was below about 20 percent, the denominator of the second term $\ln \frac{1}{1-X}$ could be approximated to $x$ by a Taylor expansion. Then the general sample size equation can be approximated as follows:

$$
n \geq(r+1) \cdot \frac{1}{x} \cdot\left(\frac{L_{B X}^{*}}{h}\right)^{\beta}+r
$$

If the acceleration factors in equation (9) were added into the planned testing time, equation (19) would be modified as:

$$
n \geq(r+1) \cdot \frac{1}{x} \cdot\left(\frac{L_{B X}^{*}}{A F \cdot h_{a}}\right)^{\beta}+r
$$

The reliability target of the pneumatic cylinder in tool changing device was guaranteed to be B1 life of 10 years. Based on the expected customer usage conditions, the normal range of operating conditions and cycles of the product (or parts) were investigated. Under the worst case, the number of required test cycles could be obtained from equation (25) for given sample pieces. ALT equipment can then be conducted on the basis of load analysis. Under different accelerated conditions, engineer could carry out parametric ALT to achieve the reliability target—a B1 life of 10 years.

Reliability testing involves analyzing time to failure of a component obtained under normal use conditions in order to quantify its life characteristics. Obtaining such life data is often difficult because the long life times of components can include. To shortly observe failures of components, procedures have been devised to accelerate their failures by overstress. Thus they fail more quickly than they would under normal use conditions. 
However, a relationship between the reliability of a component determined by ALT and its reliability at normal use conditions is necessary. This can be assessed by extrapolating the test results obtained from an accelerated life testing and comparing it to that obtained from testing at normal use conditions (see Figure 4).

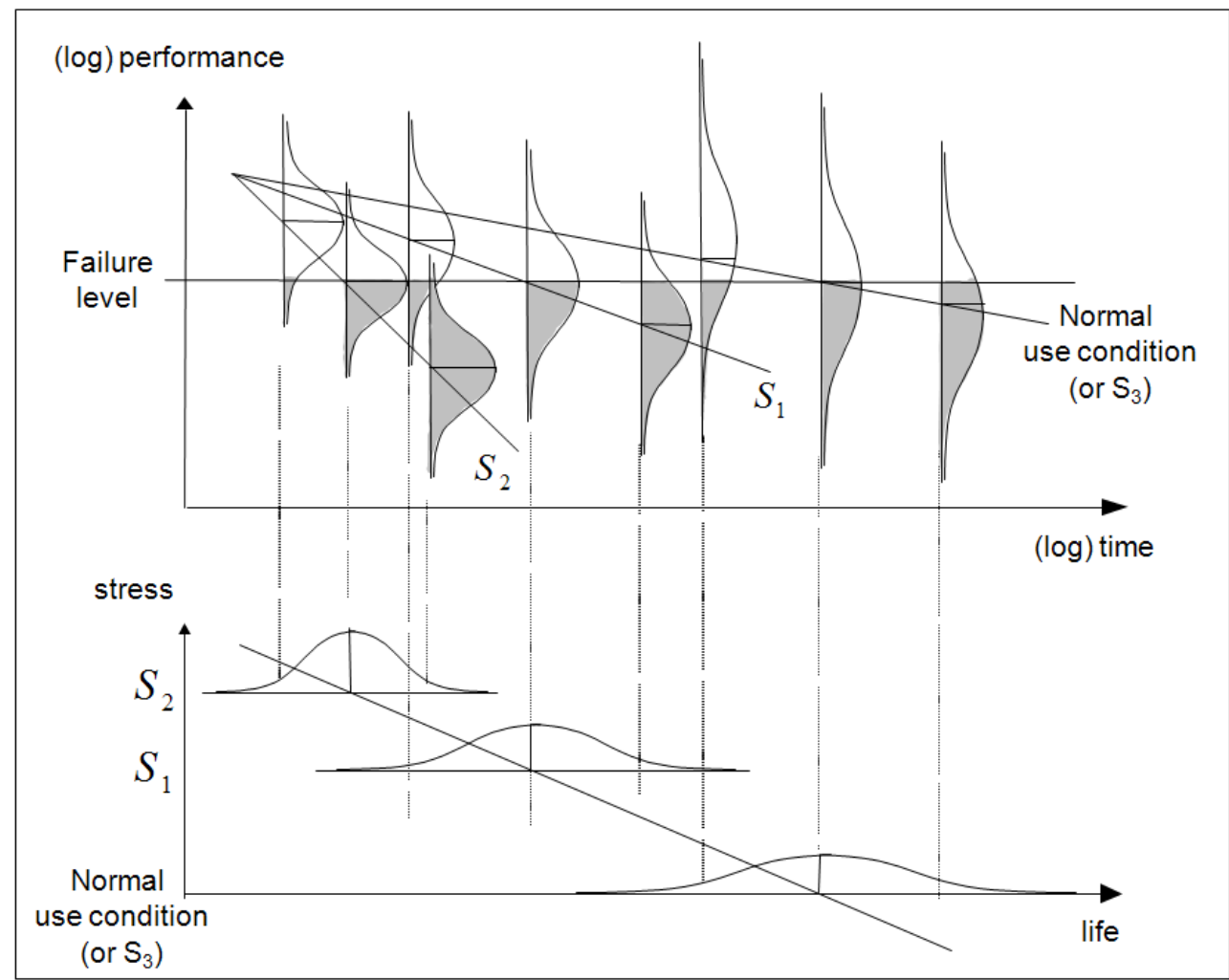

Figure 4. Relationship between S-N curve and accelerated life testing.

\section{Case Study—Lifetime of Pneumatic Cylinder}

The pneumatic cylinder produced commercially is to move a load along a linear path to the desired destination, which is normally operated at $0.63 \mathrm{MPa}, 23{ }^{\circ} \mathrm{C}$. In changing the tools, the pneumatic cylinder is subjected to repetitive pressure loads (or stress). The tool changing device in machine tools consequently depends on the lifetime of the pneumatic cylinder. It is important to replace the pneumatic cylinder after its lifetime is estimated through the proper reliability methodology—-parametric ALT. Depending on specifications of company A or B, the primary components in a pneumatic cylinder consist of seal, cylinder tube, piston rod, piston head, cap, etc. (see Figure 5 \& Table 2).

The functional loss of the pneumatic cylinder for two companies often had been reported in the manufacturing process or by owners of the machine tools. When field data for company A were comprehensively reviewed, the damaged products might have been leaked in piston. The failure mechanism is as follow: (1) subjects to the repetitive pressure loads in the pneumatic cylinder; (2) overheats of piston seal; (3) causes the hardening and wear of piston seal; and (4) exceeds Minimum Operation Pressure (MOP) and Stroke Time (ST) due to leakage [see Figure 6(a)].

On the other hand, when the field data of the damaged products for company B were investigated, the port might have blocked due to slurry mixed by oil and seal. The failure mechanism is as follow: (1) subjects to the 
repetitive pressure loads in the pneumatic cylinder; (2) causes the wear of piston seal; (3) generates the slurry and flows it into port; (4) stacks the slurry into port and blocks it; and (5) exceeds Minimum Operation Pressure (MOP) and Stroke Time (ST) due to blockage [see Figure 6(b)].

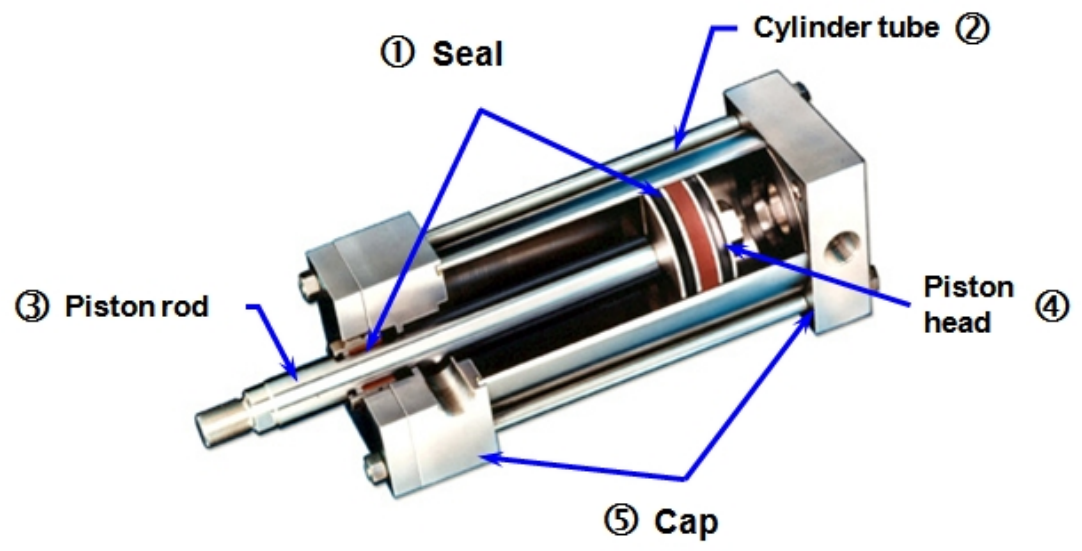

Figure 5. Mechanical parts of pneumatic cylinder: seal (1); cylinder tube (2); piston rod (3); piston head (4); and cap (5).

Table 2

Structure of Primary Components for Two Companies (A/B)

Red cap

The data of the failed products in the field were important for understanding and determining the usage patterns of consumers and helping to pinpoint design changes that needed to be implemented in the product. Based on field data, the root cause(s) of the problematic pneumatic cylinder was found. If the required reliability is targeted, we can know what component(s) in the pneumatic cylinder should be redesigned to improve reliability.

To move a load along a linear path to the desired destination, the pneumatic cylinder included several mechanical structural parts. Depending on the consumer usage conditions, the pneumatic cylinder in machine tools was often subjected to repetitive mechanical pressure loads when the tool devices change. Tool changing involved the simple mechanical processes: (1) The machine picks out the tool switched; and (2) it then replaces the required tool (see Figure 7).

To find out the required acceleration factor, it was important to understand the loads on the pneumatic cylinder during tool changing. Because the pneumatic cylinder was a relatively simple mechanical structure, the pressure in the pneumatic cylinder depends on the usage loads of end-users (see Figure 8). 


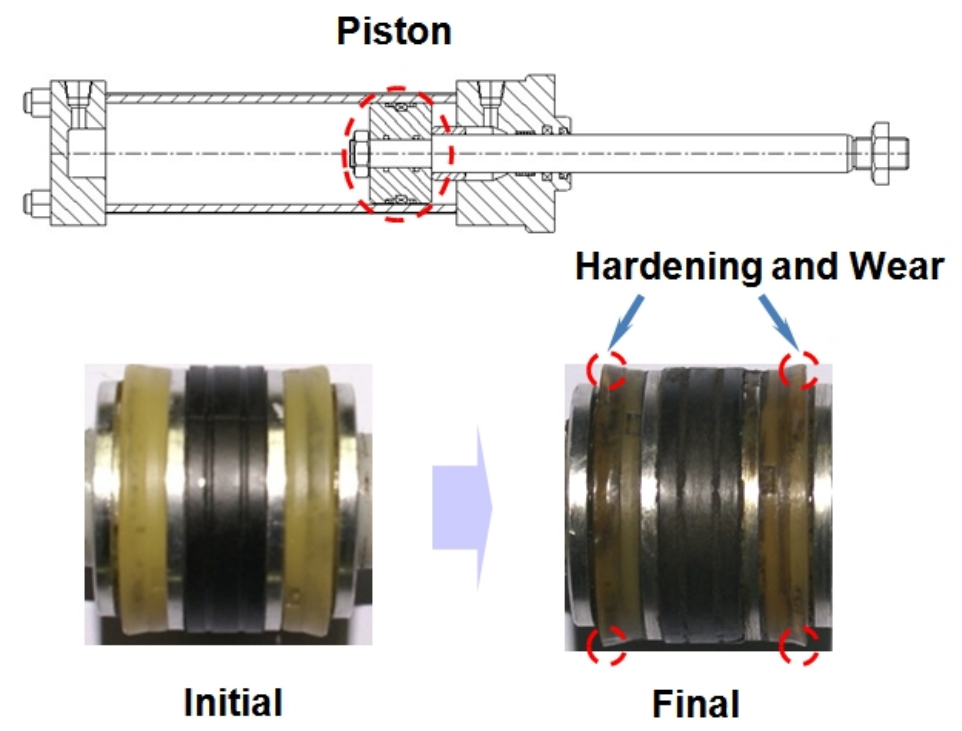

(a) Failure mode of company A.

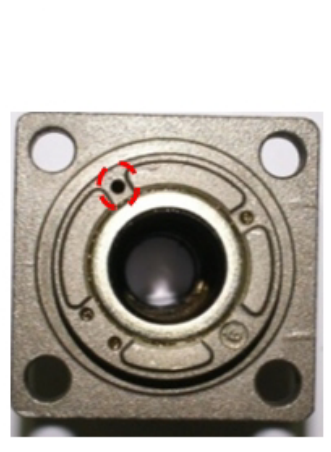

Initial

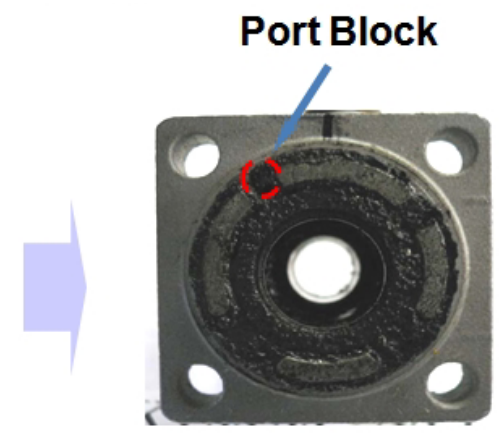

Final

(b) Failure mode of company B.

Figure 6. Example of damaged products returned from the field.

Key Noise Parameters

N1: Customer Usage \& Load Conditions

N2: Environmental Conditions

Picks out the tool
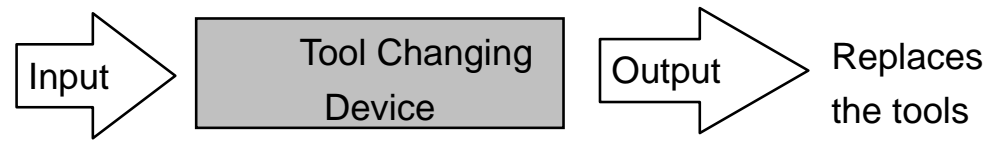

Key Control Parameters

C1: Pneumatic Cylinder

C2: Chuck material \& size

C3: Gear Box material \& size

Figure 7. Parametric design schematic of the tool changing device. 

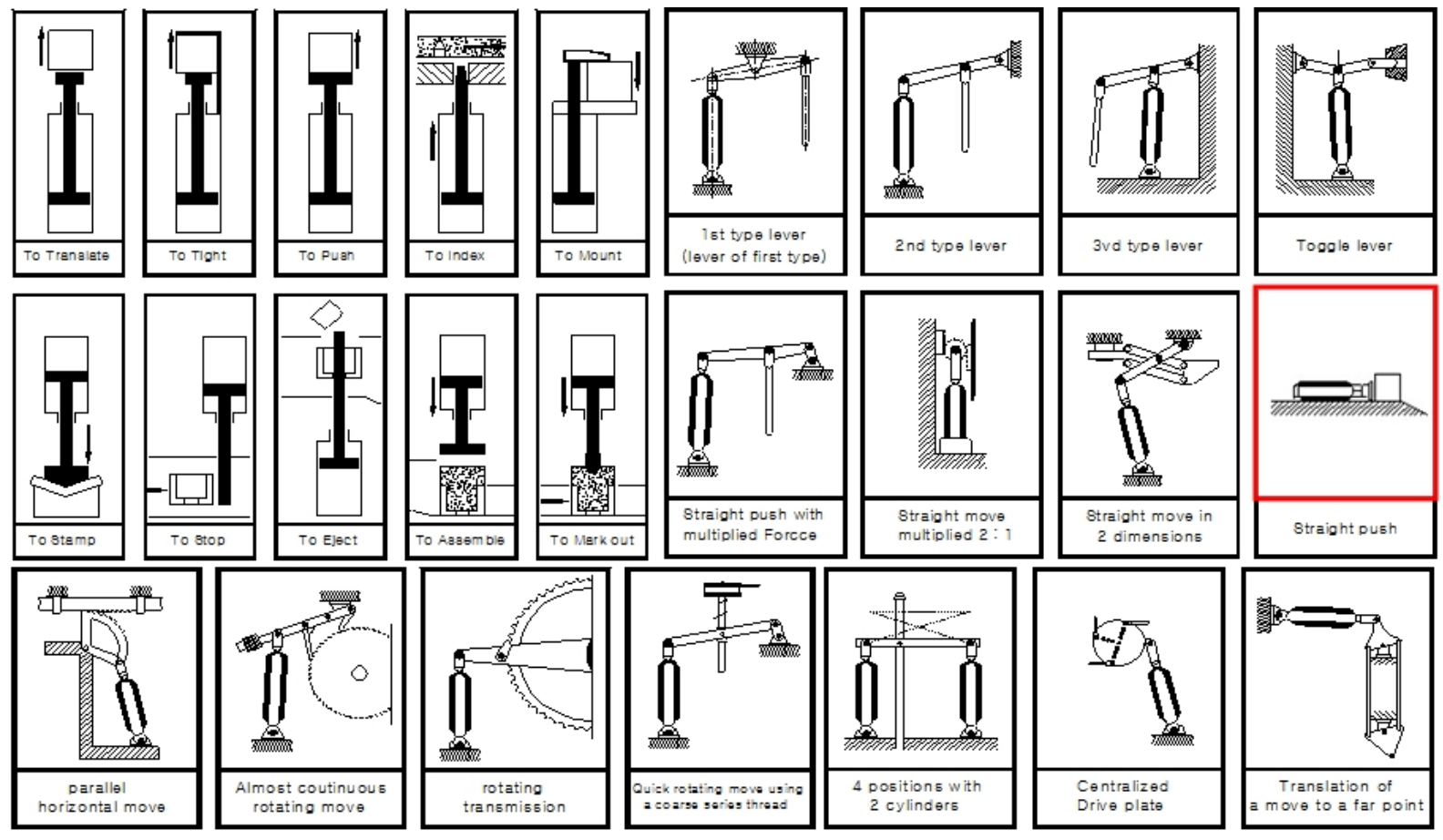

Figure 8. Pneumatic cylinder usages in field.

The pressure of the pneumatic cylinder due to external load can be simply defined as:

$$
P=F_{\text {ext }} / A
$$

The product lifetime depends on stress due to applied force that generates internal pressure in pneumatic cylinder. Under the user environmental conditions, the life-stress model in equation (8) could be modified as:

$$
T F=C(S)^{-n}=C F^{-\lambda}=C^{\prime}(P \times A)^{-\lambda}=B(P)^{-\lambda}
$$

where $\mathrm{C}, \mathrm{C}^{\prime}$, or $\mathrm{B}$ is constant.

When $P_{0}$ is the pressure in normal conditions and $P_{1}$ is the pressure in the accelerated tests, the acceleration factor (AF) in equation (9) and (27) can be simplified as:

$$
A F=\left(\frac{S_{1}}{S_{0}}\right)^{n}=\left(\frac{F_{1}}{F_{0}}\right)^{\lambda}=\left(\frac{P_{1}}{P_{0}}\right)^{\lambda}
$$

\section{Results \& Discussion}

The operating conditions for the pneumatic cylinder were approximately $0-43{ }^{\circ} \mathrm{C}$ with a relative humidity ranging from $0 \%$ to $95 \%$, and $0.2-0.24$ g's of acceleration. The tool changing in machine tools occurs in an estimated average of 10 to 30 times per day. With a life cycle design point for 10 years, the life of pneumatic cylinder incurred about 100,000 usage cycles for the worst case scenario.

To obtain the testing data at the normal condition $-23^{\circ} \mathrm{C}$ and $0.63 \mathrm{MPa}$, reliability testing was carried out for nine samples of company A. Test specifications of pneumatic cylinder are $32 \mathrm{~mm}$ (bore size) and $160 \mathrm{~mm}$ 
(stroke). To apply the loading pressure, we adjusted the lumped mass hung at end. It takes one second to complete a cycle that the pneumatic cylinder slides to and from stroke $(160 \mathrm{~mm})$.

The control panel was used to operate the testing equipment. It controlled the number of tests, the testing time, and the starting or stopping of the equipment. When the start button on the controller panel was pressed, simple pneumatic cylinder in the test equipment pushes the adjusted lumped mass and is subjected to the pressure load (0.63 MPa) (see Figure 9).

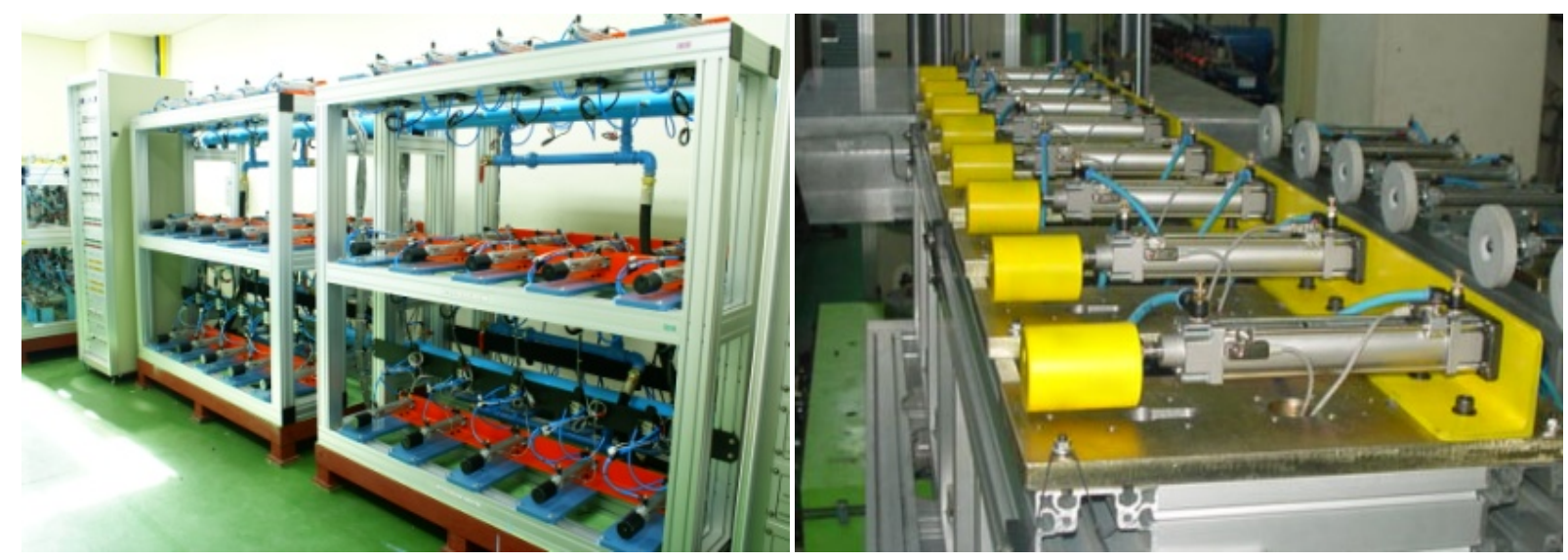

Figure 9. Equipment used in the ISO or accelerated life testing.

In result, pneumatic cylinder failed seven out of nine samples as follows: 7 million cycles (1,946 hr), 10 million cycles (2,780 hr), 12 million cycles (3,336 hr), 16 million cycles (4,448 hr), 22 million cycles (5,560 hr), and $2 \times 24$ million cycles $(6,116 \mathrm{hr})$. As seen in field, the failure mode of samples exceeded the minimum operation pressure and stroke time piston.

When the failed pneumatic cylinders were broke down, we found the hardening and wear of piston seal like that in field. The failure of the pneumatic occurs as following: (1) Piston seal is subjected to the repetitive pressure loads; (2) generates the friction heat in and vaporizes the lube in seal; (3) accelerates seal hardening and wear; and (4) leaks the seal. When the pneumatic cylinder is operated, minimum operation pressure will exceed. The shape parameter $\beta$ was determined to be 1.97 and the characteristic life $\eta$ was $2.35 \times 10^{7}$. The expected B1 life was $2.3 \times 10^{6}$.

To extrapolate the lifetime of the pneumatic cylinder for company A, two reliability tests are obtained under the accelerated conditions. Firstly, reliability testing was carried out at $0.8 \mathrm{MPa}$ and $23^{\circ} \mathrm{C}$, in which the lumped mass at end was adjusted. Using a cumulative damage exponent of 2.0, the acceleration factor was found to be approximately 1.6 in equation (28).

For the B1 life, the test cycles for 10 samples calculated in equation (25) were 200,000 cycles if the shape parameter was supposed to be 2.0. This parametric ALT was designed to ensure a B1 life of 10 years with about a $60 \%$ level of confidence that it would fail less than once during 200,000 cycles. As seen in Figure 10, repetitive stress can be expressed as the duty effect due to the on/off cycles and pneumatic cylinder shortens part life (Ajiki, Sugimoto, \& Higuchi, 1979).

In result, pneumatic cylinder failed eight out of 10 samples as follows: 6 million cycles (1,668 hr), 8 million cycles (2,224 hr), 10 million cycles (2,780 hr), 12 million cycles (3,336 hr), 16 million cycles (4,448 hr), 18 million cycles (5,004 hr), 19 million cycles (5,282 hr), and 22 million cycles $(6,116 \mathrm{hr})$. The failure mode of 
samples exceeded the minimum operation pressure and stroke time piston. When the failed pneumatic cylinders were broke down, we found the hardening and wear of piston seal like those of field samples (see Figure 11).

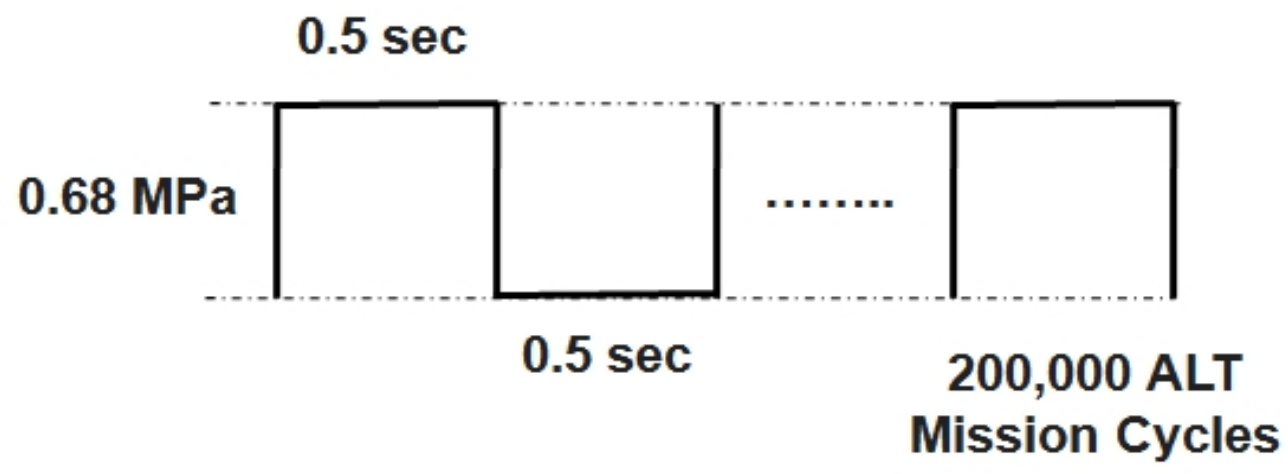

Figure 10. Duty cycles of the repetitive pressure load $\mathrm{P}$ in the pneumatic cylinder.
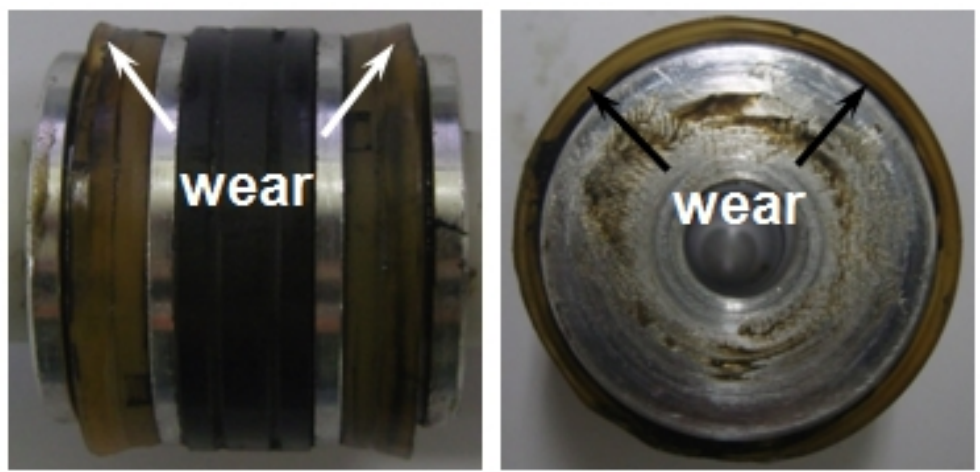

(a) Failed products in the testing data at the normal condition (or field).

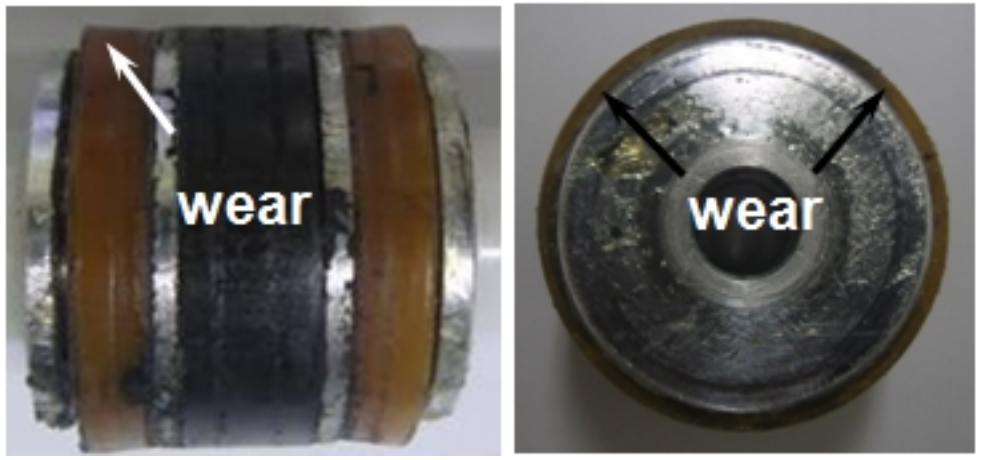

(b) Failed products after parametric ALT.

Figure 11. Failed products in field and parametric ALT.

As shown in figures, the seal shape of the parametric ALT was very similar to that of the ones from the field. Due to seal hardening and wear, we know that the pneumatic cylinder exceeded the minimum operation pressure and stroke time. Figure 12 represented the graphical analysis of the parametric ALT results and field data on a Weibull plot. As the shape parameter was initially estimated at 2.0, the shape parameter was confirmed to be 2.1 from the Weibull plot of the first ALT. These methodologies were valid in reproducing the failure mode responsible for failures in the field (or normal condition). The characteristic life $\eta$ was $1.5 \times 10^{7}$ cycles. The expected B1 life was $1.7 \times 10^{6}$ cycles. 


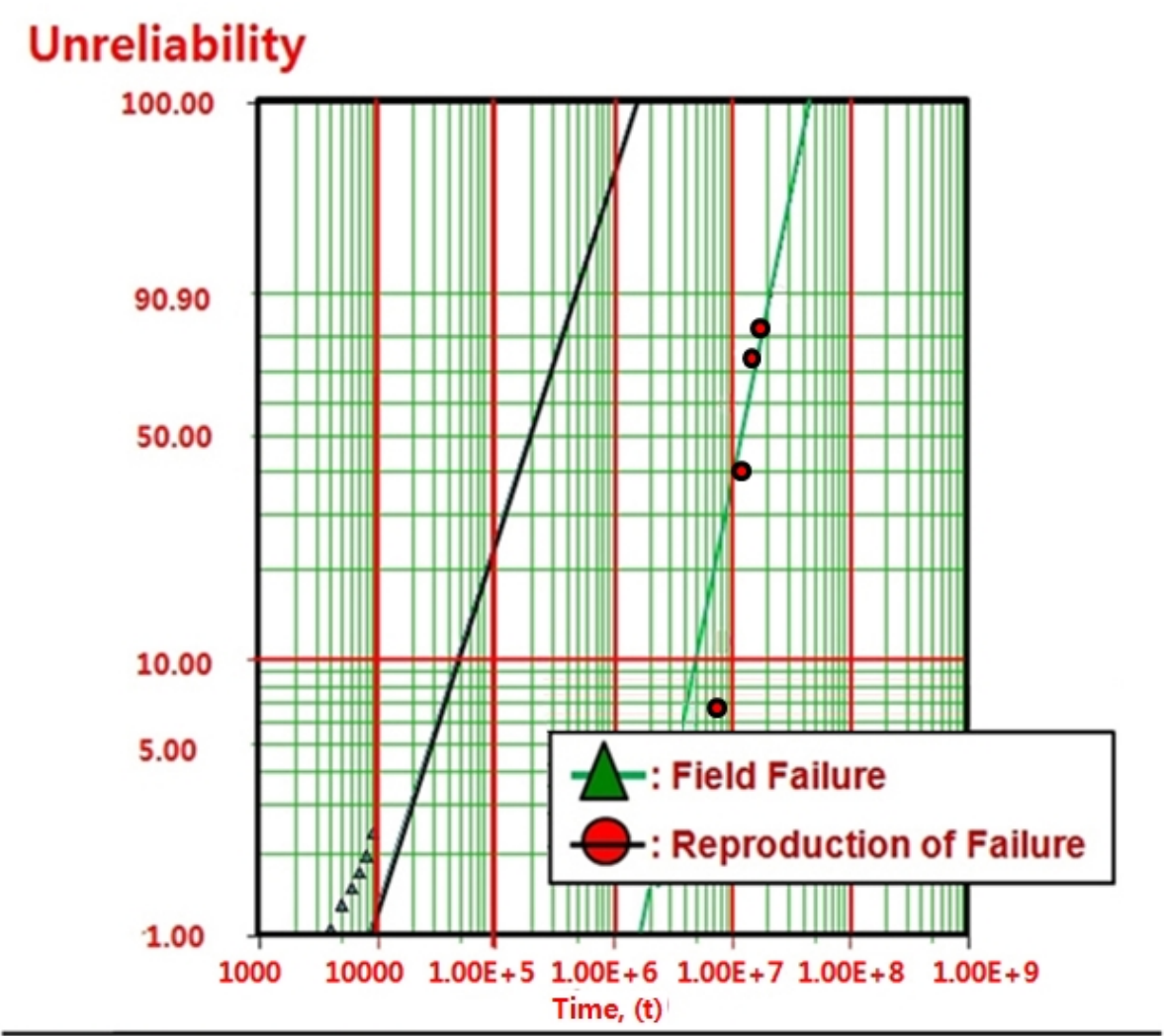

$\beta 1=2.1203, \eta=1.51 E+7$

$\beta 2=1.9660, \eta=2.52 E+5$

Figure 12. Field data and parametric ALT on Weibull chart.

To reveal the failures of the pneumatic cylinder under another accelerated condition, reliability testing was carried out at $1.2 \mathrm{MPa}$ and $23{ }^{\circ} \mathrm{C}$ as the limped mass at end was adjusted. In result, pneumatic cylinder failed five out of six samples as follows: 3.5 million cycles (972 hr), 5 million cycles (1,389 hr), 6 million cycles (1,668 hr), 8 million cycles (2,222 hr), and 10 million cycles $(2,778 \mathrm{hr})$. The failure mode of samples exceeded the minimum operation pressure and stroke time piston. When the failed pneumatic cylinders were broke down, we also found the hardening and wear of piston seal. The characteristic life $\eta$ was $8.5 \times 10^{6}$ cycles and shape parameter was 2.3. The expected B1 life was $1.15 \times 10^{6}$ cycles.

Based on the data of two parametric ALT testing, we can estimate the lifetime of pneumatic cylinder at normal use conditions. We know that B1 life is $1.73 \times 10^{6}$ cycles at $0.8 \mathrm{MPa}$ and $23^{\circ} \mathrm{C}$ and B1 life is $1.15 \times$ $10^{6}$ cycles at $1.2 \mathrm{MPa}$ and $23^{\circ} \mathrm{C}$. From Equation (27) we can get the cumulative damage exponent $(\lambda=1.0)$ and constant $\left(\mathrm{B}=1.52 \times 10^{12}\right)$. We can extrapolate $\mathrm{B} 1$ life at normal conditions- $0.63 \mathrm{MPa}$ and $23^{\circ} \mathrm{C}$, which is $2.39 \times 10^{6}$ cycles. Comparing it with test data at normal use conditions, we know that the error is less than $10 \%$.

To obtain the testing data of company $\mathrm{B}$, reliability testing at $0.63 \mathrm{MPa}$ and $23^{\circ} \mathrm{C}$ also was carried out for nine samples. In accordance with ISO 19973-3 test specifications of pneumatic cylinder are $32 \mathrm{~mm}$ (bore size) and $160 \mathrm{~mm}$ (stroke). To apply the loading pressure, we also adjusted the lumped mass hung at end. It takes one second to complete a cycle. When the start button on the controller panel was pressed, the test equipment will start. 
In result, pneumatic cylinder failed eight out of 10 samples as follows: 7 million cycles (1,946 hr), 11 million cycles (3,056 hr), $2 \times 20$ million cycles (5,555 hr), 21 million cycles (5,833 hr), 25 million cycles (6,944 hr), and $2 \times 30$ million cycles (8,333 hr). The failure mode of samples exceeded the minimum operation pressure and stroke time piston like field data.

When the failed pneumatic cylinders were broke down, we found the rod cap blocked by slurry. The failure of the pneumatic cylinder occurs as following: (1) subjects to the repetitive pressure loads in the pneumatic cylinder; (2) causes the wear of piston seal; (3) generates the slurry and flows it into port; and (4) stacks the slurry into port and blocks it. When the pneumatic cylinder is operated, minimum operation pressure will exceed. The shape parameter $\beta$ was determined to be 1.89 and the characteristic life $\eta$ was $2.92 \times 10^{7}$ cycles. The expected B1 life was $2.56 \times 10^{6}$ cycles.

To extrapolate the lifetime of the pneumatic cylinder, two reliability tests are obtained under the accelerated conditions. Firstly, reliability testing was carried out at $0.8 \mathrm{MPa}$ and $23{ }^{\circ} \mathrm{C}$ as the lumped mass at end was adjusted. This parametric ALT was designed to ensure a B1 life of 10 years with about a $60 \%$ level of confidence that it would fail less than once during 250,000 cycles.

In result, pneumatic cylinder failed eight out of 10 samples as follows: 6 million cycles (1,668 hr), $2 \times 8$ million cycles (2,224 hr), 10 million cycles (2,780 hr), 14 million cycles (3,889 hr), 17 million cycles (4,722 hr), 18 million cycles $(5,004 \mathrm{hr})$, and 20 million cycles $(5,556 \mathrm{hr})$. The failure mode of samples exceeded the minimum operation pressure and stroke time piston. When the failed pneumatic cylinders were broke down, we found the rod cap blocked by slurry like those of field samples (see Figure 13).

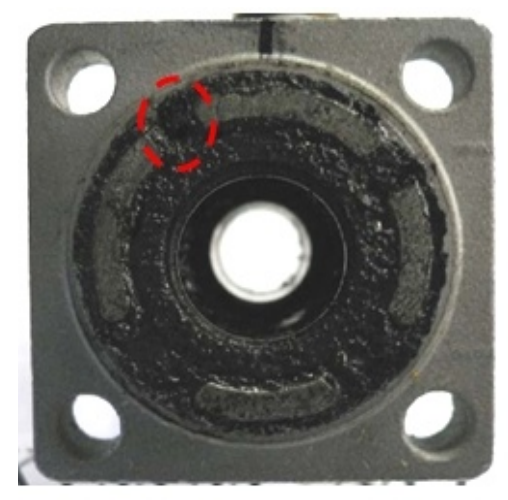

(a) Failed products in field.

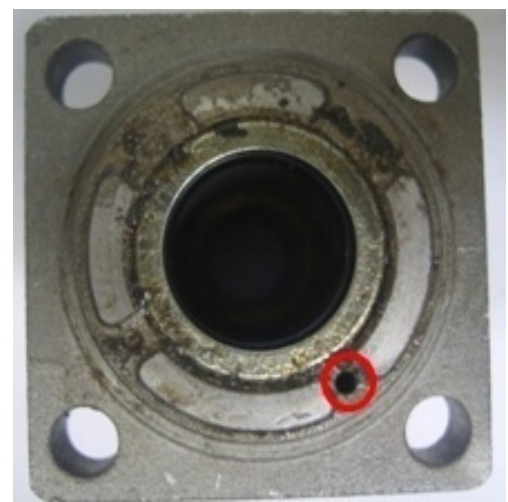

(b) Failed products after parametric ALT.

Figure 13. Failed products in field and parametric ALT.

As shown in figures, the failed rod cap shape of the parametric ALT was very similar to that of the field sample. As blocked by slurry, the pneumatic cylinder exceeded the minimum operation pressure and stroke time. The characteristic life $\eta$ was $1.7 \times 10^{7}$ cycles and the shape parameter was confirmed to be 2.1 . The expected B1 life was $1.96 \times 10^{6}$ cycles.

To reveal the failures of the pneumatic cylinder under another accelerated condition, reliability testing was carried out at $1.5 \mathrm{MPa}$ and $23{ }^{\circ} \mathrm{C}$ as the lumped mass at end was adjusted. In result, pneumatic cylinder failed three out of six samples as follows: 2.8 million cycles ( $778 \mathrm{hr}), 4$ million cycles $(1,111 \mathrm{hr})$, and 5 million cycles (1,389 hr). The failure mode of samples exceeded the minimum operation pressure and stroke time piston. When the failed pneumatic cylinders were broke down, we also found the hardening and wear of piston seal. 
The characteristic life $\eta$ was $6.2 \times 10^{6}$ cycles and shape parameter was 2.69 . The expected B1 life was $2.51 \times$ $10^{6}$ cycles.

Based on the data of two parametric ALT testing, we can estimate the lifetime of pneumatic cylinder at normal use conditions. From two accelerated testing we know that B1 life is $1.96 \times 10^{6}$ cycles at $0.8 \mathrm{MPa}$ and B1 life is $1.13 \times 10^{6}$ cycles at $1.4 \mathrm{MPa}$. From Equation (27), we can get the cumulative damage exponent $(\lambda=1.0)$ and constant $\left(B=1.40 \times 10^{12}\right)$ and extrapolate B1 life at normal conditions- $0.63 \mathrm{MPa}$ and $23^{\circ} \mathrm{C}$. So it is 2.51 $\times 10^{6}$ cycles. Comparing it with test data at normal use conditions, we know that the error is less than $10 \%$.

\section{Conclusions}

To estimate the lifetime of a newly designed mechanical component like pneumatic cylinder in machine tools, we have suggested new reliability methodologies-(1) setting overall parametric ALT plan; (2) parametric accelerated life testing with an acceleration factor and the sample size equation; and (3) as a case study, we suggest reliability design of the pneumatic cylinder.

From the testing sample of company A, we found the hardening and wear of piston seal like that of field. We can reproduce the field failure by the reliability testing. Based on two accelerated test data of products- -0.8 MPa and 1.2 MPa at $23{ }^{\circ} \mathrm{C}$, lifetime of company A can be extrapolated at normal use conditions. B1 life therefore is $2.39 \times 10^{6}$ at $0.63 \mathrm{MPa}$ and $23^{\circ} \mathrm{C}$. Comparing it with extrapolated data at normal use conditions, we know that the error is less than $10 \%$.

On the other hand, for company B, we found the rod cap blocked by slurry like those of field samples. Based on the accelerated conditions- $-0.8 \mathrm{MPa}$ and $1.4 \mathrm{MPa}$ at $23{ }^{\circ} \mathrm{C}$, we can extrapolate the lifetime of pneumatic cylinder at normal use conditions. That is, B1 life is $2.51 \times 10^{6}$ at $0.63 \mathrm{MPa}$ and $23^{\circ} \mathrm{C}$. Comparing it with test data at normal use conditions, we know that the error is less than $10 \%$.

Through the inspection of returned products in field, load analysis, and parametric ALTs for pneumatic cylinders of two companies, we can estimate their lifetime. This methodology will be helpful for engineer to know the lifetime of mechanical system like pneumatic cylinder. End-users therefore manufacture their commodity without delay due to the failures of mechanical product in field.

\section{References}

Ajiki, T., Sugimoto, M., \& Higuchi, H. (1979). A new cyclic biased THB power dissipating ICs. In 17th Annual Proceedings Reliability Physics (pp. 118-126). San Francisco, California, April 24-26, 1979.

Ben-Gal, I., Herer, Y., \& Raz, T. (2003). Self-correcting inspection procedure under inspection errors. IIE Transactions on Quality and Reliability, 34(6), 529-540.

Elsayed, E. A. (2003). Accelerated life testing. In Handbook of reliability engineering (pp. 415-428). New York: Springer-Verlag. International Organization for Standardization. (2007). ISO 19973-1: Pneumatic fluid power-Assessment of component reliability by testing-Part 1: General procedures.

Kreyszig, E. (2006). Advanced engineering mathematics (9th ed.). New Jersey: John Wiley and Son.

Karnopp, D., Margolis, D., \& Rosenberg, R. (2000). System dynamics: Modeling, simulation, and control of mechatronic systems (5th ed.). New York: John Wiley \& Sons.

Nelson, W. (1980). Accelerated life testing-Step-Stress models and data analyses. IEEE Transactions on Reliability, 29(2), 103-108.

Ryu, D., \& Chang, S. (2005). Novel concept for reliability technology. Microelectronics Reliability, 45(3), 611-622.

Spencer, F. W. (1991). Statistical methods in accelerated life testing. Technometrics, 33(3), 360-362.

Standards Coordinating Committee of the Computer Society of IEEE. (1990). IEEE standard glossary of software engineering terminology. IEEE Std. 610.12-1990. New York. 
Woo, S., \& Pecht, M. (2008). Failure analysis and redesign of a helix upper dispenser. Engineering Failure Analysis, 15(4), 642-653.

Woo, S., O’Neal, D., \& Pecht, M. (2009a). Improving the reliability of a water dispenser lever in a refrigerator subjected to repetitive stresses. Engineering Failure Analysis, 16(5), 1597-1606.

Woo, S., O’Neal, D., \& Pecht, M. (2009b). Design of a hinge kit system in a kimchi refrigerator receiving repetitive stresses. Engineering Failure Analysis, 16(5), 1655-1665.

Woo, S., O’Neal, D., \& Pecht, M. (2009c). Reliability design and case study of a refrigerator compressor subjected to repetitive loads. International Journal of Refrigeration, 32(3), 478-486.

Woo, S., O’Neal, D., \& Pecht, M. (2010a). Failure analysis and redesign of the evaporator tubing in a kimchi refrigerator. Engineering Failure Analysis, 17(2), 369-379.

Woo, S., O’Neal, D., \& Pecht, M. (2010b). Reliability design of a reciprocating compressor suction reed valve in a common refrigerator subjected to repetitive pressure loads. Engineering Failure Analysis, 17(4), 979-991.

Woo, S, O’Neal D., \& Pecht, M. (2011). Reliability design of residential sized refrigerators subjected to repetitive random vibration loads during rail transport. Engineering Failure Analysis, 18(5), 1322-1332.

Woo, S., \& O’Neal, D. (2015). Reliability design of mechanical systems subject to repetitive stresses. Recent Patents on Mechanical Engineering, 8(4), 222-234.

Woo, S. (2017). Reliability design of mechanical systems: A guide for mechanical and civil engineers (1st ed.). New York: Springer. 